\title{
Pesticide Use Practices Among Tomato Growers in Kavre District, Nepal
}

\author{
Aruna Thapa, Ananda Shova Tamrakar and Indra Prasad Subedi \\ Central Department of Zoology, TribhuvanUniversity, Kirtipur, Kathmandu, Nepal
}

\begin{abstract}
Common practices to manage the pests of tomato were explored in three VDCs (Panchkhal, Nala and Mahadevsthan) of Kavre District from January to April 2011. The data were collected by direct observation and also by using semi-structured questionnaires. Altogether 48 tomato farms were observed during the study period that included 16 farms in each VDC. Farmers were found to be relied on pesticides from bedding of plant till harvesting to control pests. Farmers were found positive towards the pesticide use despite their familiarity on ill-effects of pesticides. Personal safety measures during application of pesticides have not been followed. Scanty knowledge, labor intensiveness and lack of coordination between the farmers were the major factors which prevented the adoption of eco-friendly alternative pest management methods.
\end{abstract}

Key words: Aphis, Bemisia, Helicoverpa, IPM, White grub

\section{Introduction}

The tomato (Lycopersicon esculentum Mill.) is herbaceous fruiting plant that survives in diverse environmental conditions (Rice et al. 1987). Tomato fruit is considered to be fairly rich in vitamins of high cash value and used in our daily life for various purposes but farmers are using pesticides to kill pests and diseases which attack them for better production (Knezevic and Serdar 2008). Over 1000 compounds are applied to agricultural crops in order to control objectionable moulds, insects and weeds (Ortelli et al. 2006). Pesticides' striking effort in preventing crop loss and controlling vectors of diseases have led to their acceptance and expanded use throughout the world (Sharpdan and Peter 2005). However, the potent chemicals for killing pests have elevated anxiety that they are agents of human diseases and environmental pollution. It has been observed that their long term, low dose exposure is increasingly linked to human health effects such as immune-suppression, hormone disruption, diminished intelligence, reproductive abnormalities and cancer (Wiles et al. 1998). Pesticide residues in food are global problems (Abinash and Singh 2009). According to WHO, developing countries use about twenty-five percent of the pesticides in the world and the use is in increasing trend (WHO 2003). This intrinsically dangerous technology is being promoted in a setting without technical and human resources to control it properly.

Agriculture is one of the most prevalent types of employment in the world. Nearly 50 percent of the world labor is employed in agriculture and they carry significant risk for development of pesticide risk (Das et al. 2001). Chemical pesticides for the first time were commenced in Nepal in 1952 when Paris green, Gammexane and Nicotine sulphate were imported from USA solely for malaria control program (Koirala et al. 2009). The sequential order of different groups of pesticides introduced in Nepal is: 1950s organochlorines; 1960s - organophosphates; 1970s - carbamates; 1980s - synthetic pyrethroids (Manandhar 2007).

Nepalese Journal of Zoology | Online Volume 3 Issue 1 | November 2015 | Page 17 
There is no clear drift of import and use of pesticides. Also there is possibility of an open border trading of commonly used pesticides and some of the banned pesticides such as DDT and BHC. It is somewhat difficult to document the amount of illegal trading and thus the size of such trading has not been reflected in the public data so far. In recent years, there are different pesticides used erratically, which is pretty common in Nepal. Endosulfan is used in ponds, streams and rivers for killing fishes and also used to attain polished appearance in vegetables such as tomato, brinjal, mustard leaves (Manandhar 2007).

Nepal has a marvelous opportunity for producing tomato as diverse agro-eco-zone favors both season and off-season varieties and farmers are encouraged to produce, so production and productivity has been increasing significantly for the last decade. Nepal has potential for fresh vegetable (tomato) and processed products in the international market as well. Reports suggested that use of pesticides in vegetables in Nepal is higher than other food products (Koirala et al. 2009). Therefore, this study aims to know the current situation of pesticide used in tomato and to deal the knowledge, practice among tomato growers and use of pesticides in Kavre district, Nepal.

\section{Materials and Methods}

Insect pests of tomato and their management practices were explored in three VDCs of Kavre District (Panchkhal, Nala and Mahadevsthan). These are major tomato production areas in the district so were chosen for this study which was conducted from January to April 2011. The data were collected by direct observation and also by using semi-structured questionnaires. Altogether 48 tomato farms were observed during the study that included 16 farms in each VDC. Tomato field was visited for direct observation of the plants and insect pests. The information regarding pesticide use was collected from farmers, agro-vet dealers, government agencies and related associations.

\section{Results}

\section{Opinion of farmers on application of pesticide in the field}

Majority of farmers (79.2\%) of the study area had positive opinion towards the use of pesticide. The farmers' views on the increased use of pesticides in the field include proper and safe for health and environment, increases crop production and reduces loss due to pest damage. They further mentioned that the knowledge, awareness and training about pesticides is a must. About $20.8 \%$ farmers were with negative opinion on the use of pesticides. They mentioned that the use of pesticides must be decreased in the field and biofirendly alternatives must be implemented to prevent health and environmental hazard.

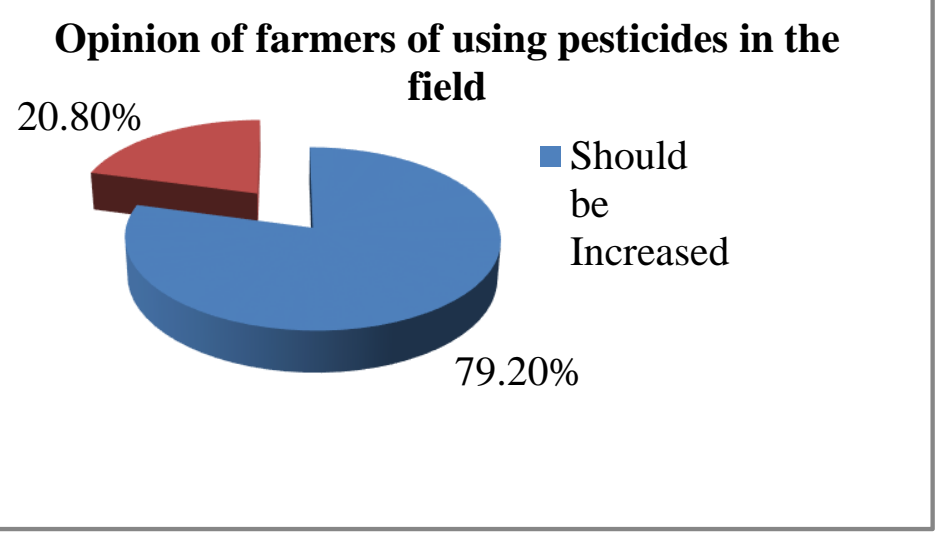

Figure 1. Opinion of farmers of using pesticides in the field

Nepalese Journal of Zoology | Online Volume 3 Issue 1 | November 2015 | Page 18 


\section{Use of Pesticides for Pest Control}

A total of 48 farmers and their fields were surveyed during the study. All of respondents (100\%) were reported to use pesticides in tomatoes as pest control measure. It indicates that there is a high frequency of pesticides use in the vegetables which pose higher risk to vegetable growers and consumers.

Table 1. Pesticide users among tomato growers

\begin{tabular}{|l|l|}
\hline Pesticide user among tomato growers & Percentage \\
\hline Yes- use pesticides & $100 \%$ \\
\hline No- do not use pesticides & $0 \%$ \\
\hline
\end{tabular}

\section{Knowledge and practices regarding use of pesticides}

During study $80 \%$ farmers were found to use pesticides to increase productivity, $18.5 \%$ of them used pesticides to kill pest and $1.5 \%$ used to improve color. About $24.8 \%$ determine amount of pesticides to be used according to the information given in the label, $60.2 \%$ determine through retailer where they purchase the pesticide and $15 \%$ determine with their local agricultural expert. About $95.8 \%$ farmers bought pesticides from authorized shop or retailers whereas $4.2 \%$ from non-authorized shops.

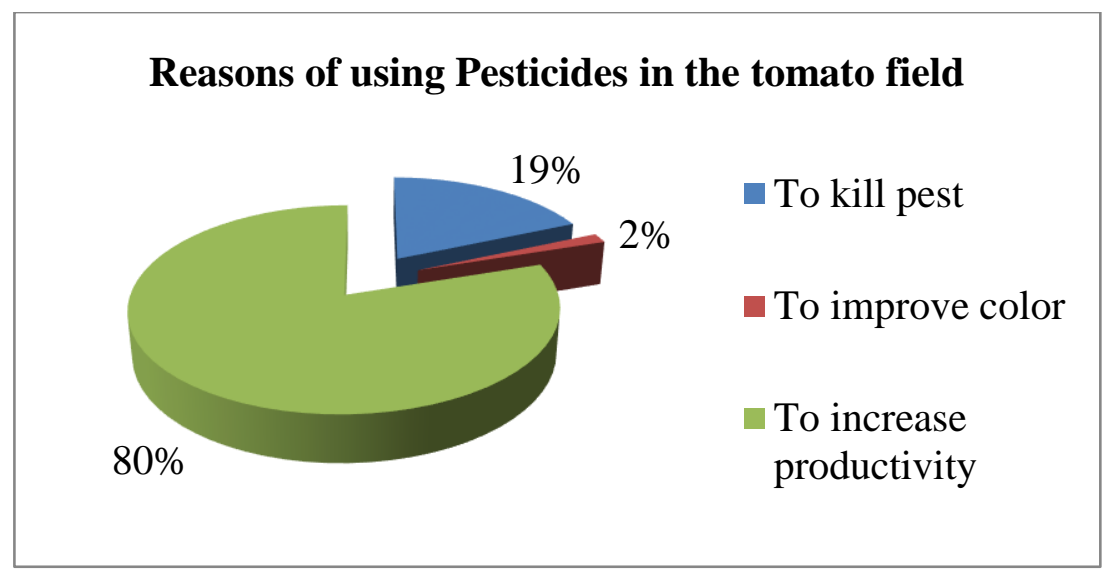

Figure 2. Reasons of using Pesticides in the tomato field

\section{Safety precautions, personal protective equipment and personal hygiene during application of pesticides}

Majority of respondent farmers (91.6\%) read the label in the bottle or package where as $8.3 \%$ didn't read the label. About $70.8 \%$ mixed the pesticides with hands wearing hand gloves, $4.2 \%$ mixed with bare hands and $25 \%$ mixed with stick and wearing hand gloves. During study, we found that none of them eat, drink or smoke while spraying pesticides in the field. Most of them, 50\% washed the pesticide bottle or pesticide sprayer in the river, pond or well, whereas $45.8 \%$ washed in a distant place far from pond, river or well and $4.2 \%$ wash in tap at home. None of them display a signboard or red flag or empty bottles in the sprayed area after an application in order to inform others and also none of them keep the pesticides in the same place where they keep their food.

Nepalese Journal of Zoology | Online Volume 3 Issue 1 | November 2015 | Page 19 
Table 2. Use of Personal Protective Equipments

\begin{tabular}{|l|l|l|}
\hline Items & Yes (\%) & No (\%) \\
\hline Shoes & $100 \%$ & - \\
\hline Hat/Head cover & $33.4 \%$ & $66.6 \%$ \\
\hline Glass & $4.2 \%$ & $95.8 \%$ \\
\hline Full sleeves shirt/ trouser & $100 \%$ & - \\
\hline Gloves & $4.2 \%$ & $95.8 \%$ \\
\hline Mask & - & $100 \%$ \\
\hline Change the clothes & $100 \%$ & - \\
\hline Take a bath & $25 \%$ & $75 \%$ \\
\hline Clean hands and foots & $75 \%$ & $25 \%$ \\
\hline
\end{tabular}

\section{Disposal techniques}

About $58.3 \%$ of the farmers disposed the pesticide bottles or packets by throwing in the field, $29.2 \%$ bury in the field and $12.5 \%$ disposed in other ways like burning or use for household purpose after washing or throw them in to the dumping sites. If pesticide was spilled in some places, all of them were found that they clean the places with water only but they did not use any cleaning agent. During the application of pesticides, $87.5 \%$ had experience of pesticide spoil in their body parts and $12.5 \%$ had no such experience.

\section{Health Problems due to use of pesticides}

All the farmers (100\%) knew about the adverse effects of pesticides to human health. Out of them $75 \%$ knew that pesticide use can cause short term health effect and only $25 \%$ knew that pesticides can also cause long term health effects. The different types of health hazards experienced by the farmers due to the use of pesticides were eye irritation, vomiting, skin irritation, dizziness, headache, diarrhea and shortness to breathe. The symptoms of health effects they suffered due to the use or exposure of pesticides were determined by the health worker (37.5\%), self by the farmers (50\%) and neighbor $(12.5 \%)$ but not by the doctor.

\section{Knowledge about Integrated Pest Management (IPM)}

During the study, $96 \%$ farmers were found to be familiar with IPM where as $4 \%$ of them were infamiliar. The reasons behind not using the IPM techniques despite their familiarity include pesticides are cheaper $(62.5 \%)$, use of IPM is highly labor intensive (98.4\%), not practiced by neighbor (12.5\%) and lack of IPM training $(16.6 \%)$.

Nepalese Journal of Zoology | Online Volume 3 Issue 1 | November 2015 | Page 20 


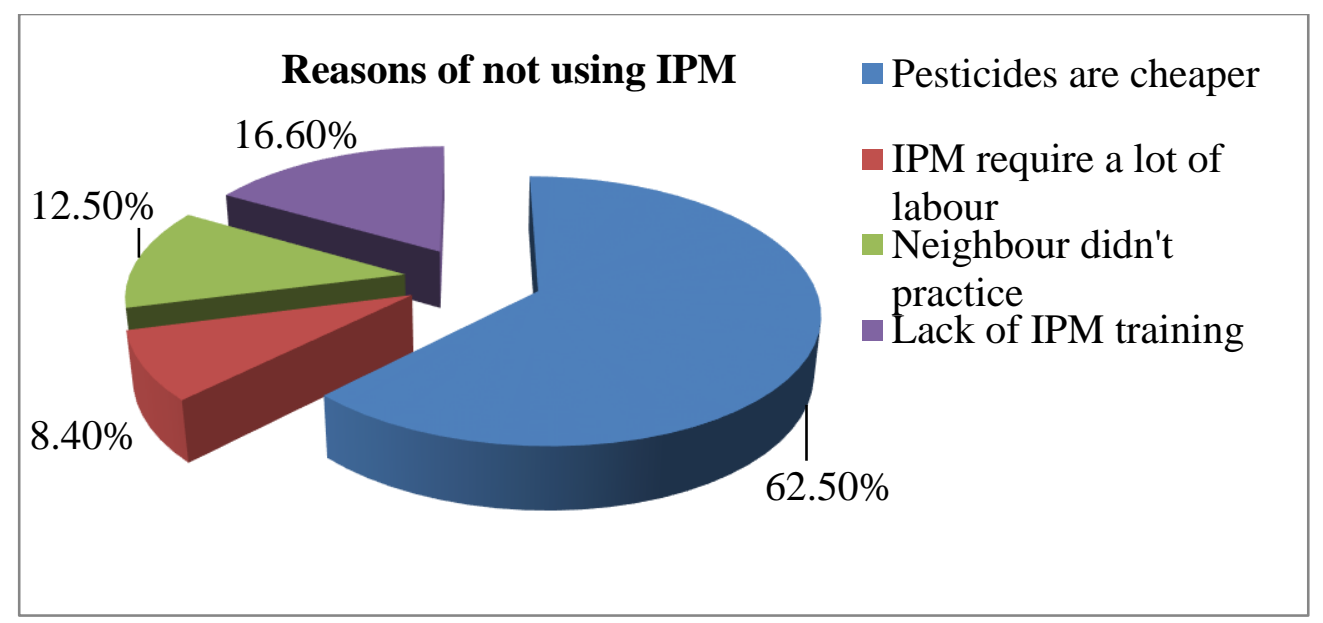

Figure 3. Reasons behind not practicing IPM techniques by farmers

\section{Discussion}

As the farming of tomato were done totally by the use of pesticides. Farmers used pesticides to increase productivity and to kill the pests so that more profit and high yield can be achieved. Even though they bought the pesticides directly from shop or agro-vet dealer and used that according to the label in containers or according to shopkeeper. Lack of knowledge about pesticides, its composition and its formulation made more misuse of pesticides. Palikhe (2002) documented that misuse and overuse of pesticides, particularly among commercial farmers, posed a health risk to the public and have numerous cases caused serious poisoning. The illegitimate used was due to unawareness of toxicity, aggressive marketing by dealers and profit interests. Many farmers did not understand the instructions written on the pesticide labels. The harmful effects of pesticides have been experienced by farmers and their families. There was a higher risk of presence of pesticides residue in vegetables which poses higher health risk to vegetable growers as well as consumers.

The adoption of safety measures during and after pesticide application was very important factor for preventing against harmful impacts of pesticide. The various safety options could be used as gloves, masks, long sleeved cloth, glass, long boots etc. They did not like to use safety measure as they thought that they disturb their work and uncomfortable to work in the field. For example, they thought that wearing a mask makes breathing difficult and they didn't have habit of wearing (Palikhe 2002). This might put them in the risk of pesticides that the health of these people was not secured of the disease caused by the inhalation, ingestion and absorption via skin (Karmacharya 2012). Personal protective devices were also found used less because of lack of awareness, unavailable and feeling discomfort by using them. From the field study, as expressed by farmers themselves, it was difficult for them to change their behavior in which they were adopted. Due to unsafe practices, farmers were more vulnerable to expose with toxic pesticides and were in higher health risks as there has been too much use of pesticides with too little or no protection. This result is also supported by Shrestha (2001).

Farmers knew about health effects and they suffered by different kind of symptoms. Most of the farmers knew about short term effects of pesticides where as only some knew about long term effects of pesticides (Shrestha et al. 2010). The different health problems they suffered were eye irritation, skin irritation, dizziness, shortness to breathe, vomiting, headache and diarrhea. They even diagnosed their health effects direct by themselves or by their neighbors but very few by the help of health workers. They rarely

Nepalese Journal of Zoology | Online Volume 3 Issue 1 | November 2015 | Page 21 
consulted health personnel and took treatment in health centers, and they used their home treatment by themselves, which was comparable with the research work carried out by Koirala et al. (2009).

Pesticides application in the field is not only the solution of pests control and high yield of crops besides these famers need awareness programs, training and proper guidance for application of pesticides. Other alternative methods of pests management is the demand of today. Shrestha (2001) reported that overuse of synthetic pesticides has also resulted in pest resistance to pesticides, resurgence of pests, elimination of natural enemies and disruption of ecosystems. Although the agricultural policies during the last few decades promoting higher input of chemicals have resulted in higher yields and more food, they have also resulted in poisoning, health related poverty and environmental degradation. There is, therefore, a need for alternative pest control measures for both commercial farmers currently overusing pesticides and food insecure subsistence farmers living at the mercy of pests. A healthy, effective and lasting mechanism for plant protection is required for food security, food safety, poverty reduction and rural development (Shrestha et al. 2010).

\section{Acknowledgements}

Authors are grateful to Mr Pramod Koirala, Senior Food Research officer, Department of Food Technology and Quality Control for his support and suggestions.

\section{References}

Abinash, P. C. and Singh, N. 2009. Pesticide use and application: An Indian scenario. J. Hazard Master, 165(1-3): $1-12$

Das, R., Steege, A., Baron, S., Beckman, J. and Harrison, R. 2001. Pesticide related illness among migrant farm workers in the United States Int J. Occup Environ Health Oct-Dec 7(4): 303-12

Karmacharya, S. 2012. Pesticide Use In Agriculture And Its Socio- Economic Contexts, A Case Study of Panchkhal Area, Kavre, Nepal. International journal of Science and Technology Research Nepal. 1.

Knezevic, Z. and Serdar, M. 2008. Screening of fresh fruits and vegetables for pesticide residues on Croatian Market. J. Food Control 10: 10-16

Koirala, P., Dhakal, S. and Tamrakar, A.S. 2009. Pesticides and Food Safety Issues in Nepal. The Journal of Agriculture and Environment, 10: 33-36

Manadhar, D.N. 2007. Pesticides in Nepal. SD Manandhar Publisher, Kathmandu.

Ortelli, D., Edder, P. and Corvi, C. 2006. Multi residue analysis of 74 pesticides in fruits and vegetable by liquid chromatography-electro spray-tandem ass spectrometry. Analtica Chimica Acta, 520: 33-45

Palihke, B.R. 2002. Challenges and options of pesticide use: In the context of Nepal. Plant Protection Directorate, Department of Agriculture, Harihar Bhawan, Lalitpur, Kathmandu, Nepal

Rice, R. P., Rice, L. W., and Tindall, H. D. 1987. Fruit and Vegetable Production in Africa. Macmillan Publishers, U.K. P. 371

Sharpdan, S. and Peter, C. 2005. Delayed health hazard of pesticides exposure. Annual review of public health, 27: 312-316

Shrestha, K. K. 2001. Pesticide Management Program: In view of RENAO activities. Project Management Committee and Tripartite Review Meeting on the RENAP, 9-10 July 2000. Nantong, China.

Nepalese Journal of Zoology | Online Volume 3 Issue 1 | November 2015 | Page 22 
Shrestha, P., Koirala, P. and Tamrakar, A. S. 2010. Knowledge, Practice And Use of Pesticides among Comercials Vegetables Growers Of Dhading District, Nepal. The Journal of Agriculture and Environment: 11.

WHO, 2003. Pesticide residue in food. In: International program on chemical safety Joint FAO/WHO meeting on pesticides results evaluations 2002, part 2 Toxicology.

Wiles, R., Davies, K. and Campbell, C. 1998. Over exposed organophosphate insecticides in children's food. Environmental working group, Washington.

Nepalese Journal of Zoology | Online Volume 3 Issue 1 | November 2015 | Page 23 